\title{
Exponent Preserving Subgroups of the Finite Simple Groups
}

\author{
Andrea Pachera \\ pak.ska@gmail.com
}

August 13, 2018

\begin{abstract}
Given a group $G$ denote with $\exp (G)$ its exponent, which is the least common multiple of the order of its elements. In this paper we solve the problem of finding the finite simple groups having a proper subgroup with the same exponent. For each $G$ with this property we will give an explicit example of $H<G$ with $\exp (G)=\exp (H)$.
\end{abstract}

\section{Introduction}

Given a group $G$, denote with $\pi(G)$ the set of prime divisors of $|G|$, with $\Gamma(G)$ its prime graph, and with $\exp (G)$ its exponent, i.e. the least common multiple of the orders of its elements.

In the recent years a series of problems have been investigated, related to the existence of a suitable subgroup $H<G$ preserving some prescribed property of $G$. For example, Lucchini, Morigi, and Shumyatsky [6] proved that if $G$ is finite then it always contains a 2-generated subgroup $H$ with $\pi(G)=\pi(H)$, and a 3-generated subgroup $H$ with $\Gamma(G)=\Gamma(H)$; Covato [3] extended these results to profinite groups; Burness and Covato [1] showed which finite simple groups $G$ contain a proper subgroup $H$ with $\Gamma(G)=\Gamma(H)$.

The aim of this work is to find the finite simple groups which have a proper subgroup with the same exponent, and we prove the following result:

Theorem. The finite simple groups which contain a proper subgroup with the same exponent are the following:

(i) the alternating groups $A_{n}$ with $n \geqslant 5$, except when $n=10, n=p^{r}$ with $p$ odd prime, or $n=p_{f}+1$ where $p_{f}$ is a Fermat prime;

(ii) the symplectic groups $\operatorname{PSp}_{4}(q)$, except when $q=3^{k}$ or $q=2$;

(iii) the symplectic groups $\operatorname{PSp}_{2 m}(q)$ with $m$ and $q$ even, except when $m=q=2$;

(iv) the orthogonal groups $\mathrm{P} \Omega_{2 m+1}(q)$ with $m \geqslant 4$ even, except when $p^{a}=2 m-1$ for some $a$, where $q=p^{k}$;

(v) the orthogonal groups $\mathrm{P} \Omega_{2 m}^{+}(q)$ with $m \geqslant 4$ even;

(vi) the Mathieu groups $\mathrm{M}_{12}, \mathrm{M}_{24}$;

(vii) the Higman-Sims group $H S$.

The proof is based on [5], where Table 10.7 can be used to obtain Table 1 below, which contains a list of all the possible pairs $(G, M)$ where $M$ is a maximal subgroup of the finite simple group $G$ with $\pi(M)=\pi(G)$. For each of these pairs $(G, M)$ we check whether $\exp (M)=\exp (G)$, organizing our discussion in the following way:

(i) in section 3 we solve the problem for the four infinite families of classical groups of Lie type (a)-(d). Notice that this requires the study of the Sylow $p$-subgroups of the groups involved, which are discussed in section 2, in order to compare the exponents of $G$ and $M$; 
(ii) the alternating group (e) is studied in section 4 ;

(iii) in the other cases, the possibilities for $M$ are explicitly listed, so it's easy to make a direct computation: they are studied in section 5 .

\begin{tabular}{|c|c|c|c|}
\hline & $G$ & $M$ & Remarks \\
\hline (a) & $\operatorname{PSp}_{2 m}(q)$ & $M \unrhd \Omega_{2 m}^{-}(q)$ & $m, q$ even \\
\hline (b) & $\mathrm{P} \Omega_{2 m+1}(q)$ & $M \unrhd \Omega_{2 m}^{-}(q)$ & $m$ even, $q$ odd \\
\hline (c) & $\mathrm{P} \Omega_{2 m}^{+}(q)$ & $M \unrhd \Omega_{2 m-1}(q)$ & $m$ even \\
\hline (d) & $\mathrm{PSp}_{4}(q)$ & $M \unrhd \operatorname{PSp}_{2}\left(q^{2}\right)$ & \\
\hline (e) & $A_{c}$ & $A_{k} \unlhd \bar{M} \leqslant S_{k} \times S_{c-k}$ & \\
\hline & $A_{6}$ & $\mathrm{~L}_{2}(5)$ & \\
\hline & $\mathrm{L}_{6}(2)$ & $P_{1}, P_{5}$ & \\
\hline & $\mathrm{U}_{3}(3)$ & $\mathrm{L}_{2}(7)$ & \\
\hline & $\mathrm{U}_{3}(5)$ & $A_{7}$ & \\
\hline & $\mathrm{U}_{4}(2)$ & $2^{4} \rtimes A_{5}, S_{6}$ & \\
\hline & $\mathrm{U}_{4}(3)$ & $\mathrm{L}_{3}(4), A_{7}$ & \\
\hline & $\mathrm{U}_{5}(2)$ & $\mathrm{L}_{2}(11)$ & \\
\hline & $\mathrm{U}_{6}(2)$ & $\mathrm{M}_{22}$ & \\
\hline & $\mathrm{PSp}_{4}(7)$ & $A_{7}$ & \\
\hline & $\operatorname{Sp}_{6}(2)$ & $S_{8}$ & \\
\hline & $\mathrm{P} \Omega_{8}^{+}(2)$ & $P_{1}, P_{3}, P_{4}, A_{9}$ & \\
\hline & $G_{2}(3)$ & $\mathrm{L}_{2}(13)$ & \\
\hline & ${ }^{2} F_{4}(2)^{\prime}$ & $\mathrm{L}_{2}(25)$ & \\
\hline & $\mathrm{M}_{11}$ & $\mathrm{~L}_{2}(11)$ & \\
\hline & $\mathrm{M}_{12}$ & $\mathrm{M}_{11}, \mathrm{~L}_{2}(11)$ & \\
\hline & $\mathrm{M}_{24}$ & $\mathrm{M}_{23}$ & \\
\hline & $\mathrm{HS}$ & $\mathrm{M}_{22}$ & \\
\hline & $\mathrm{McL}$ & $\mathrm{M}_{22}$ & \\
\hline & $\mathrm{Co}_{2}$ & $\mathrm{M}_{23}$ & \\
\hline & $\mathrm{CO}_{3}$ & $\mathrm{M}_{23}$ & \\
\hline
\end{tabular}

Table 1: The pairs $(G, M)$ with $\pi(G)=\pi(M)$.

Notation. The notation is fairly standard, with the classical groups denoted in the following way:

$$
\begin{aligned}
\mathrm{L}_{n}(q)= & \operatorname{PSL}_{n}(q) \leqslant \mathrm{SL}_{n}(q) \leqslant \mathrm{GL}_{n}(q) \\
& \operatorname{PSp}_{2 n}(q) \leqslant \operatorname{Sp}_{2 n}(q) \\
\mathrm{P} \Omega_{n}^{\eta}(q) \leqslant & \Omega_{n}^{\eta}(q) \leqslant \operatorname{SO}_{n}^{\eta}(q) \leqslant \operatorname{GO}_{n}^{\eta}(q)
\end{aligned}
$$

where $\eta= \pm 1$ if $n$ is even, and it's omitted if $n$ is odd. It may be omitted also when referring to an unspecified orthogonal group.

$\mathrm{K}_{n}(q)$ denotes the kernel of the spinor norm in $\mathrm{GO}_{n}(q)$, so that $\mathrm{K}_{n}(q) \cap \mathrm{SO}_{n}(q)=\Omega_{n}(q)$.

$P_{i}$ denotes a parabolic subgroup stabilizing a $i$-dimensional subspace.

$A \imath_{\text {tw }} B$ denotes a twisted wreath product as described in [10].

$\operatorname{ord}_{p}(q)$ denotes the multiplicative order, i.e. it's the minimum $e$ such that $q^{e} \equiv 1 \bmod p$.

$a^{b} \| c$ means that $a^{b} \mid c$ but $a^{b+1} \nmid c$.

\section{The Sylow $p$-subgroups of the classical groups}

The four infinite families to study involve the groups $\operatorname{PSp}_{2 m}(q), \mathrm{P}_{2 m+1}(q), \mathrm{P} \Omega_{2 m}^{+}(q)$, and $\Omega_{2 m}^{-}(q)$.

In order to evaluate their exponents, we study their Sylow $p$-subgroups to get the corresponding power of $p$ in the factorization of the exponent. In particular, the following hold: 
Proposition 2.1. Take $G=\operatorname{Sp}_{2 c}(q)$ or $\mathrm{GO}_{d}(q)$, $p$ odd with $p \nmid q$, $e=\operatorname{ord}_{p}(q)$, and $p^{r} \| q^{e}-1$. A Sylow p-subgroup of $G$ is isomorphic to a Sylow p-subgroup of $\mathrm{GL}_{n}(q)$ (if e is even) or $\operatorname{Sp}_{2 n}(q)$ (if $e$ is odd) for some $n$. In particular:

$$
\begin{array}{rlrl}
\exp _{p}\left(\mathrm{GL}_{n}(q)\right) & =p^{r+v} & e p^{v} \leqslant n<e p^{v+1} \\
\exp _{p}\left(\operatorname{Sp}_{2 n}(q)\right)=p^{r+v} & 2 e p^{v} \leqslant 2 n<2 e p^{v+1}
\end{array}
$$

Since $p$ is odd, then $\exp _{p}\left(\operatorname{PSp}_{2 c}(q)\right)=\exp _{p}\left(\operatorname{Sp}_{2 c}(q)\right)$ and $\exp _{p}\left(\mathrm{P}_{d}(q)\right)=\exp _{p}\left(\mathrm{GO}_{d}(q)\right)$.

Proposition 2.2. Take $q$ odd, s such that $2^{s+1} \| q^{2}-1$, and $r_{t}$ such that $2^{r_{t}} \leqslant n<2^{r_{t}+1}$, where $n=2 m$ or $n=2 m+1$ is the degree of the group. Then:

$$
\begin{aligned}
\exp _{2}\left(\mathrm{PSp}_{2 m}(q)\right) & = \begin{cases}2^{s+r_{t}-1} & \text { if } m \neq 2^{k} \\
2^{s+r_{t}-2} & \text { if } m=2^{k}\end{cases} \\
\exp _{2}\left(\Omega_{2 m+1}(q)\right)=\exp _{2}\left(\mathrm{P} \Omega_{2 m+1}(q)\right) & = \begin{cases}2^{s+r_{t}-1} & \text { if } m \neq 2^{k} \\
2^{s+r_{t}-2} & \text { if } m=2^{k}\end{cases} \\
\exp _{2}\left(\Omega_{2 m}^{\eta}(q)\right)=\exp _{2}\left(\mathrm{P} \Omega_{2 m}^{\eta}(q)\right) & =\left\{\begin{array}{ll}
2^{s+r_{t}-1} & \text { if } m \neq 2^{k} \\
2^{s+r_{t}-2} & \text { if } m=2^{k}
\end{array} \quad(m>2)\right.
\end{aligned}
$$

Proposition 2.3. Take $p \mid q$, and $G$ a symplectic or orthogonal group over the field $\mathbb{F}_{q}$. Then

$$
\exp _{p}(G)=\min \left\{p^{a} \mid p^{a}>c-1\right\}
$$

where $c$ is $2 m$ if $G=(\mathrm{P}) \operatorname{Sp}_{2 m}(q)$ or $(\mathrm{P}) \Omega_{2 m+1}(q)$, and $2 m-2$ if $G=(\mathrm{P}) \Omega_{\frac{2}{2 m}}^{ \pm}(q)$.

These calculations follow from a series of results describing the Sylow $p$-subgroups of the classical groups, proved in $[2,8,9,10]$, which we recall here.

\subsection{Sylow $p$-subgroups in characteristic prime to $p$}

The Sylow $p$-subgroups with $q$ prime to $p$ and $p \neq 2$, are described in [9]. The construction holds for $\operatorname{Sp}_{2 m}(q)$ and $\mathrm{SO}_{n}(q)$.

For the next results, take $e=\operatorname{ord}_{p}(q)$, and define $r$ as $q^{e}-1=p^{r} x$, where $(p, x)=1$.

\subsubsection{General Linear Group}

We need some information concerning the Sylow $p$-subgroups of the general linear group, since they are required in the description of the Sylow $p$-subgroups of the symplectic and orthogonal groups.

Consider $\mathrm{GL}_{n}(q)$. Suppose $n=c+e a$ and $a=a_{0}+a_{1} p+\ldots+a_{v} p^{v}$, where $0 \leqslant c<e$ and $0 \leqslant a_{i}<p$, and take $G_{0}$ a Sylow $p$-subgroup of $\mathrm{GL}_{e}(q)$, which is a cyclic group of order $p^{r}$.

For example, fix a base of $\mathbb{F}_{q^{e}}$ over $\mathbb{F}_{q}$, and identify $\operatorname{Aut}_{\mathbb{F}_{q}} \mathbb{F}_{q^{e}}$ with $\operatorname{GL}_{e}(q)$. The natural action of $\mathbb{F}_{q^{e}}^{\times}$on $\mathbb{F}_{q^{e}}$ induces an embedding $\mathbb{F}_{q^{e}}^{\times} \hookrightarrow \mathrm{GL}_{e}(q)$, then take a maximal $p$-subgroup.

Define $G_{i+1}=G_{i} \nmid C_{p}$. Then a Sylow $p$-subgroup of $\mathrm{GL}_{n}(q)$ is isomorphic to $\prod_{0}^{v} G_{i}^{a_{i}}$.

In particular, $\exp _{p}\left(\mathrm{GL}_{n}(q)\right)=\exp \left(G_{v}\right)=p^{r+v}$, where $e p^{v} \leqslant n<e p^{v+1}$.

\subsubsection{Symplectic Group}

Consider $\operatorname{Sp}_{2 n}(q)$. If $e$ is even, a Sylow $p$-subgroup of $\operatorname{Sp}_{2 n}(q)$ is already a Sylow $p$-subgroup of $\mathrm{GL}_{2 n}(q)$. If $e$ is odd, a similar construction gives that a Sylow $p$-subgroup is isomorphic to $\prod_{0}^{v} G_{i}^{b_{i}}$, where $2 n=d+2 b e(0 \leqslant d<2 e), b=b_{0}+b_{1} p+\ldots+b_{v} p^{v}\left(0 \leqslant b_{i}<p\right), G_{0}$ is a Sylow $p$-subgroup of $\operatorname{Sp}_{2 e}(q)$, and $G_{i+1}=G_{i} \prec C_{p}$.

$G_{0}$ is again a cyclic group of order $p^{r}$ : consider the subgroup $R$ of $\operatorname{Sp}_{2 e}(q)$ of all $M=\left(\begin{array}{cc}A & 0 \\ 0 & B\end{array}\right)$ where $A$ and $B$ belong to a Sylow $p$-subgroup of $\mathrm{GL}_{e}(q)$. Being symplectic implies that $A^{\top} B=1$, so that $R$ is isomorphic to a Sylow $p$-subgroup of $\mathrm{GL}_{e}(q)$.

In particular, $\exp _{p}\left(\operatorname{Sp}_{2 n}(q)\right)=\exp \left(G_{v}\right)=p^{r+v}$, where $e p^{v} \leqslant 2 n<e p^{v+1}$ if $e$ is even and $2 e p^{v} \leqslant 2 n<2 e p^{v+1}$ if $e$ is odd. 


\subsubsection{Orthogonal Group}

In odd dimension, the construction is almost the same as for the symplectic group (notice that $\mathrm{Sp}_{2 m}(q)$ and $\mathrm{GO}_{2 m+1}(q)$ have the same order), giving the same result in term of exponent evaluation: $\exp _{p}\left(\mathrm{SO}_{2 m+1}(q)\right)=p^{r+v}$, where $e p^{v} \leqslant 2 m+1<e p^{v+1}$ if $e$ is even and $2 e p^{v} \leqslant 2 m<2 e p^{v+1}$ if $e$ is odd. In even dimension, consider $\mathrm{SO}_{2 m}^{\varepsilon}(q)$ : a Sylow $p$-subgroup is already a Sylow $p$-subgroup of $\mathrm{SO}_{2 m+1}(q)$ if $p \mid q^{m}-\varepsilon$, and a Sylow $p$-subgroup of $\mathrm{SO}_{2 m-1}(q)$ otherwise.

\subsection{Sylow 2-subgroups in odd characteristic}

The construction of Sylow 2-subgroups is described in [2] and [10]. The idea is the same as before, with proper adjustments.

\subsubsection{Symplectic Group}

Consider $\operatorname{Sp}_{2}(q)$ first, and denote with $W$ a Sylow 2-subgroup. Since $\left|\operatorname{Sp}_{2}(q)\right|=q\left(q^{2}-1\right),|W|=$ $2^{s+1}$ where $2^{s+1} \| q^{2}-1$.

If $q \equiv 1 \bmod 4$, let $\varepsilon$ be a primitive $2^{s}$-th root of unity in $\mathbb{F}_{q}$. Then

$$
W \simeq\left\langle\left(\begin{array}{cc}
\varepsilon & 0 \\
0 & \varepsilon^{-1}
\end{array}\right),\left(\begin{array}{cc}
0 & 1 \\
-1 & 0
\end{array}\right)\right\rangle .
$$

If $q \equiv 3 \bmod 4$, let $\varepsilon$ be a primitive $2^{s+1}$-th root of unity in $\mathbb{F}_{q^{2}}$. Then

$$
W \simeq\left\langle\left(\begin{array}{cc}
0 & 1 \\
1 & \varepsilon+\varepsilon^{q}
\end{array}\right)^{2},\left(\begin{array}{cc}
0 & 1 \\
-1 & 0
\end{array}\right)\right\rangle .
$$

By writing $W \simeq\langle X, Y\rangle$, it's clear that $\exp (W)=\exp (X)=2^{s}$.

Theorem 2.4. Let $S$ be a Sylow 2-subgroup of $\operatorname{Sp}_{2 n}(q)$, and write $2 n=2^{r_{1}}+\ldots+2^{r_{t}}$, where $r_{1}<\ldots<r_{t}$. Then $S \simeq W_{r_{1}} \times \ldots \times W_{r_{t}}$, where $W_{r}=W<\underbrace{C_{2} \imath \ldots \succ C_{2}}_{r-1}$.

In particular, $\exp _{2}\left(\operatorname{Sp}_{2 n}(q)\right)=\exp \left(W_{r_{t}}\right)=2^{s+r_{t}-1}$.

A Sylow 2-subgroup $S^{\prime}$ of $\operatorname{PSp}_{2 n}(q)$ is obtained by quotienting a Sylow 2-subgroup $S$ of $\operatorname{Sp}_{2 n}(q)$ over $S \cap Z$ where $Z=\left\langle-1_{n}\right\rangle$ is the centre of the group.

If $S \simeq W_{r_{1}} \times \ldots \times W_{r_{t}}$, then there exists an element of the form $\left(1_{2^{r_{1}}}, \ldots, 1_{2^{r_{t-1}}}, g\right)$ of maximum order, whose powers meet the centre only in $1_{n}$.

If $S \simeq W_{r}$, notice that an element $x \in W_{r}=W_{r-1} \prec C_{2}$ with maximum order $2^{c}$ is of the form $((g, h), \sigma)$, where $g h \in W_{r-1}$ has maximum order and $C_{2}=\langle\sigma\rangle$, so that $x^{2^{c-1}}=$ $\left(\left(-1_{2^{r-1}},-1_{2^{r-1}}\right), 1\right)=-1_{2^{r}}$.

Therefore,

$$
\exp _{2}\left(\operatorname{PSp}_{2 n}(q)\right)= \begin{cases}2^{s+r_{t}-1} & \text { if } n \neq 2^{k} \\ 2^{s+r_{t}-2} & \text { if } n=2^{k}\end{cases}
$$

where $2^{s+1} \| q^{2}-1$, and $2^{r_{t}} \leqslant 2 n<2^{r_{t}+1}$.

\subsubsection{Orthogonal Group}

Consider the groups $\mathrm{SO}_{2 n+1}(q)$ and $\mathrm{GO}_{2 n}^{ \pm}(q)$ first.

Since $\left|\mathrm{GO}_{2}^{\varepsilon}(q)\right|=2(q-\varepsilon)$, again $|W|=2^{s+1}$ where $2^{s+1} \| q^{2}-1$. $W$ is dihedral so $\exp (W)=2^{s}$, in particular we may take $W=\langle u, w\rangle$, where:

- if $q \equiv 1 \bmod 4$, then the underlying form is of plus type and is represented by $Q\left(x_{1}, x_{2}\right)=$ $2 x_{1} x_{2}$. Take $\varepsilon$ a primitive $2^{s}$-th root of unity in $\mathbb{F}_{q}$ and

$$
u=\left(\begin{array}{cc}
\varepsilon & 0 \\
0 & \varepsilon^{-1}
\end{array}\right) \quad w=\left(\begin{array}{cc}
0 & 1 \\
1 & 0
\end{array}\right)
$$


- if $q \equiv 3 \bmod 4$, then the underlying form is of minus type and is represented by $Q\left(x_{1}, x_{2}\right)=$ $x_{1}^{2}+x_{2}^{2}$. Take $a, b \in \mathbb{F}_{q}$ such that $a+b \sqrt{-1}$ is a primitive $2^{s}$-th root of unity in $\mathbb{F}_{q^{2}}$ and

$$
u=\left(\begin{array}{cc}
a & b \\
-b & a
\end{array}\right) \quad w=\left(\begin{array}{cc}
-1 & 0 \\
0 & 1
\end{array}\right) .
$$

An order comparison shows that a Sylow 2-subgroup of $\mathrm{SO}_{3}(q)$ may be obtained with the natural embedding $W \mapsto\left(\begin{array}{cc}\operatorname{det} W & 0 \\ 0 & W\end{array}\right)$. In general, the following holds:

Theorem 2.5. Let $S$ be a Sylow 2-subgroup of $\mathrm{SO}_{2 n+1}(q)$ and let $2 n=2^{r_{1}}+\ldots+2^{r_{t}}$, with $r_{1}<\ldots<r_{t}$. Then $S \simeq W_{r_{1}} \times \ldots \times W_{r_{t}}$, where $W_{r}=W \imath \underbrace{C_{2}} \imath \ldots \imath C_{2}$ and $W$ is a Sylow 2-subgroup of $\mathrm{GO}_{2}^{\eta}(q)$ with $q \equiv \eta \bmod 4$.

While in even dimension:

Theorem 2.6. Let $S$ be a Sylow 2-subgroup of $\mathrm{GO}_{2 n}^{\eta}(q)$.

(i) If $q^{n} \equiv \eta \bmod 4$, then $S$ is isomorphic to a Sylow 2-subgroup of $\mathrm{SO}_{2 n+1}(q)$.

(ii) If $q^{n} \equiv-\eta \bmod 4$, then $S \simeq C_{2} \times C_{2} \times S_{0}$, where $S_{0}$ is a Sylow 2-subgroup of $\operatorname{SO}_{2 n-1}(q)$.

The exponent of a Sylow 2-subgroup is the same in $\mathrm{GO}_{2 n}(q)$ and $\mathrm{SO}_{2 n}(q)$. The argument is similar to the one used to deduce (7): consider the previous construction $W=\langle u, w\rangle$ and check the determinant. If $n=2^{k}$, a Sylow 2-subgroup of $\mathrm{SO}_{2}(q)$ is obtained using $W^{\prime}=\langle u\rangle$ instead, which has the order halved but the same exponent. If $n \neq 2^{k}$, consider a Sylow 2-subgroup $\left(C_{2} \times C_{2}\right) \times W_{r_{1}} \times \ldots \times W_{r_{t}}$ of $\mathrm{GO}_{2 n}(q)$, then an element of maximum order and determinant one can be obtained by taking an element of $W_{r_{t}}$ with maximum order, and adjusting the determinant by taking suitable elements of the other groups.

The comparison of the exponents of $\mathrm{SO}_{n}(q)$ and $\Omega_{n}(q)$ shares the same idea, i.e. if $n \neq 2^{k}$ then the exponent doesn't change: $S \simeq\left(C_{2} \times C_{2}\right) \times W_{r_{1}} \times \ldots \times \ldots W_{r_{t}}$ so take an element of $W_{r_{t}}$ with maximum order, and adjust the spinor norm taking a proper element of the other groups.

It's not immediately obvious what happens when $n=2^{r}$ though, i.e. if there exists an element of maximum order and spinor norm 1 . The result can be deduced anyway using theorems $7,8,10$ in [10].

Theorem 2.7. If $q^{n} \equiv-\eta \bmod 4$, then a Sylow 2-subgroup of $\Omega_{2 n}^{\eta}(q)=\mathrm{P} \Omega_{2 n}^{\eta}(q)$ is isomorphic to a Sylow 2-subgroup of $\mathrm{GO}_{2 n-2}^{\eta^{\prime}}(q)$, where $q^{n-1} \equiv \eta^{\prime} \bmod 4$.

Theorem 2.8. A Sylow 2-subgroup of $\Omega_{2 n+1}(q)=\mathrm{P} \Omega_{2 n+1}(q)$ is isomorphic to a Sylow 2-subgroup of $\mathrm{K}_{2 n}^{\eta}(q)$, where $q^{n} \equiv \eta \bmod 4$.

So the remaining cases are $\mathrm{K}_{2 n}^{\eta}(q)$ and $\Omega_{2 n}^{\eta}(q)$, where $q^{n} \equiv \eta \bmod 4$ and $n=2^{r}$.

Consider $\mathrm{K}_{2 n}^{\eta}(q)$ first. Take $n=1$, and remember the construction of a Sylow 2-subgroup of $\mathrm{GO}_{2}(q)$ as $W \simeq\langle u, w\rangle$, with $u^{2^{s}}=w^{2}=1$ and $u^{w}=u^{-1}(2.2 .2)$.

The Sylow 2-subgroup of $\mathrm{K}_{2}(q)$ contained in $W \simeq\langle u, w\rangle$ is $W^{\prime} \simeq\langle v, w\rangle$, where $v=u^{2}$. It's still dihedral, but it has both the order and the exponent halved, being now $2^{s}$ and $2^{s-1}$ respectively. In particular:

$$
v^{2^{s-1}}=w^{2}=1, \quad v^{w}=v^{-1} .
$$

Put $e=u w$, so that $e \in \mathrm{GO}_{2}(q) \backslash \mathrm{K}_{2}(q), e^{2}=u u^{w}=1$ and $W \simeq W^{\prime}\langle e\rangle$, where

$$
v^{e}=v^{-1}, \quad w^{e}=v w .
$$

From now on use $e$ to denote $\left(\begin{array}{cc}e & 0 \\ 0 & 1_{2 n-2}\end{array}\right) \in \mathrm{GO}_{2 n}(q) \backslash \mathrm{K}_{2 n}(q)$, which has order 2 .

Theorem 2.9. Take $\mathrm{K}_{2 n}^{\eta}(q)$, where $n=2^{r}(r \geqslant 0)$ and $q^{n} \equiv \eta \bmod 4$. Let $2^{s+1} \| q^{2}-1$, $E=\langle e\rangle \simeq C_{2}, W^{\prime} \simeq\langle v, w\rangle$ as in (8), $\rho: E \rightarrow$ Aut $W^{\prime}$ as in (9), and $V=\langle a, b\rangle \simeq C_{2} \times C_{2}$.

Then a Sylow 2-subgroup of $\mathrm{K}_{2 n}^{\eta}(q)$ is isomorphic to the repeated twisted wreath product $W_{r}^{\prime}=$ $W^{\prime} \imath_{t w} \underbrace{V \imath_{t w} \cdots \imath_{t w} V}_{r}$, where the action of $V$ on $\left(W_{i}^{\prime}\right)^{2}$ is given by $(x, y)^{a}=\left(x^{e}, y^{e}\right)$ and $(x, y)^{b}=$ $(y, x)$ for any $x, y \in W_{i}^{\prime}$. 
With this action, $\exp \left(W_{r}^{\prime}\right)=2 \cdot \exp \left(W_{r-1}^{\prime}\right)$, so that $\exp \left(W_{r}^{\prime}\right)=2^{r} \exp \left(W^{\prime}\right)=2^{r+s-1}$. Indeed, take for example $v_{i} \in W_{i}^{\prime}$ of maximum order, then $\left(\left(v_{i}, 1\right), b\right) \in W_{i+1}^{\prime}$ has double its order.

Consider now $\Omega_{2 n}^{\eta}(q)$ and $\mathrm{P} \Omega_{2 n}^{\eta}(q)=\Omega_{2 n}(q) / Z$, where $Z=\langle z\rangle$, and $z=-1_{2 n}$ is the centre of $\Omega_{2 n}^{\eta}(q)$, and again $q^{n} \equiv \eta \bmod 4$.

For $n=1$ a Sylow 2-subgroup of $\Omega_{2}^{\eta}(q)$ is $W^{\prime \prime}=\langle v\rangle$, which has order and exponent $2^{s-1}$. This time, $W$ is not a split extension of $T$, so the inductive construction has to start from $n=2$.

Consider again $e=\left(\begin{array}{cc}u w & 0 \\ 0 & 1_{2 n-2}\end{array}\right) \in \mathrm{GO}_{2 n}^{\eta}(q) \backslash \mathrm{K}_{2 n}^{\eta}(q)$, and take

$$
f=\left(\begin{array}{ccc}
1_{2} & 0 & 0 \\
0 & w & 0 \\
0 & 0 & 1_{2 n-4}
\end{array}\right) \in \mathrm{K}_{2 n}^{\eta}(q) \backslash \Omega_{2 n}^{\eta}(q) .
$$

$F=\langle e, f\rangle$ is then a non-cyclic group of order 4 with trivial intersection with $\Omega_{2 n}(q)$. For $n=2$, one may take $W^{\prime \prime}=\langle d, g, h, k\rangle$, where

$$
d=\left(\begin{array}{cc}
u & 0 \\
0 & u^{-1}
\end{array}\right), \quad g=\left(\begin{array}{cc}
u & 0 \\
0 & u
\end{array}\right), \quad h=\left(\begin{array}{cc}
0 & 1_{2} \\
1_{2} & 0
\end{array}\right), \quad k=\left(\begin{array}{cc}
0 & w \\
w & 0
\end{array}\right) .
$$

In particular,

$$
\begin{gathered}
d^{2^{s-1}}=g^{2^{s-1}}=z, \quad z^{2}=h^{2}=k^{2}=1, \quad d^{h}=d^{-1}, \quad g^{k}=g^{-1}, \\
{[d, g]=[d, k]=[h, g]=[h, k]=1 .}
\end{gathered}
$$

which means that $W^{\prime \prime}$ is a central product of two dihedral groups of order $2^{s+1}$, and $\langle z\rangle$ is the centre of $W^{\prime \prime}$. Then, the action of $F$ on $W^{\prime \prime}$ is given by

$$
\begin{gathered}
d^{e}=g^{-1}, \quad g^{e}=d^{-1}, \quad h^{e}=g k, \quad k^{e}=d h, \\
d^{f}=g, \quad g^{f}=d, \quad h^{f}=k, \quad k^{f}=h .
\end{gathered}
$$

Theorem 2.10. Take $\Omega_{2 n}^{\eta}(q)$, where $n=2^{r}(r \geqslant 1)$ and $q^{n} \equiv \eta \bmod 4$. Let $2^{s+1} \| q^{2}-1$, $F=\langle e, f\rangle \simeq C_{2} \times C_{2}, W^{\prime \prime} \simeq\langle d, g, h, k\rangle$ as in (10), $\rho: F \rightarrow$ Aut $W^{\prime \prime}$ as in (11), and $V=\langle a, b, c\rangle \simeq$ $C_{2} \times C_{2} \times C_{2}$.

Then a Sylow 2-subgroup of $\Omega_{2 n}^{\eta}(q)$ is isomorphic to the repeated twisted wreath product $W_{r}^{\prime \prime}=$ $W^{\prime \prime} \imath_{t w} \underbrace{V 2_{t w} \cdots \imath_{t w} V}_{r-1}$, where the action of $V$ on $\left(W_{i}^{\prime \prime}\right)^{2}$ is given by $(x, y)^{a}=\left(x^{e}, y^{e}\right),(x, y)^{b}=$ $\left(x^{f}, y^{f}\right)$, and $(x, y)^{c}=(y, x)$ for any $x, y \in W_{i}^{\prime \prime}$.

Again $\exp \left(W_{r}^{\prime \prime}\right)=2 \cdot \exp \left(W_{r-1}^{\prime \prime}\right)$, so the exponent is $\exp \left(W_{r}^{\prime \prime}\right)=2^{r-1} \exp \left(W^{\prime \prime}\right)=2^{r+s-2}$.

Here, passing from $\Omega_{2 n}(q)$ to $\mathrm{P} \Omega_{2 n}(q)$ requires to replace $W^{\prime \prime}$ with $W^{\prime \prime} /\langle z\rangle$ in the construction. This lowers the exponent when $r=1$, but it's irrelevant since $\mathrm{P} \Omega_{4}^{+}(q)$ is not simple and $\mathrm{P} \Omega_{4}^{-}(q) \simeq$ $\operatorname{PSL}_{2}\left(q^{2}\right)$ is already studied elsewhere. For $r>1$ the exponent doesn't change, take for example $r=2: d$ and $g$ have order $2^{s-1}$ since in the projective group $z=1$.

Then $x=((d, 1), a c) \in\left(W^{\prime \prime} /\langle z\rangle\right)_{2}$ has order $2^{s}$, indeed:

$$
\begin{aligned}
x & =((d, 1), a c), \\
x^{2} & =\left((d, 1)\left(1, d^{e}\right), 1\right)=\left(\left(d, g^{-1}\right), 1\right), \\
x^{3} & =\left((d, 1)\left(g^{-e}, d^{e}\right), a c\right)=\left(\left(d^{2}, g^{-1}\right), a c\right), \\
x^{4} & =\left((d, 1)\left(g^{-e},\left(d^{e}\right)^{2}\right), 1\right)=\left(\left(d^{2}, g^{-2}\right), 1\right), \\
x^{2^{s-1}} & =\left(\left(d^{2^{s-2}}, g^{-2^{s-2}}\right), 1\right), \\
x^{2^{s}} & =\left(\left(d^{2^{s-1}}, g^{-2^{s-1}}\right), 1\right)=((1,1), 1) .
\end{aligned}
$$




\subsection{Sylow $p$-subgroups in characteristic $p$}

This case is completely solved by corollary 0.5 of [8], which in our situation can be simplified in the following way:

Theorem 2.11. Let $G$ be a classical group defined over a field of characteristic $p$. Then the exponent of a Sylow p-subgroup of $G$ is $\min \left\{p^{a} \mid p^{a}>c-1\right\}$, where $c$ is the Coxeter number of $G$.

Note that the original proof of this theorem depends upon the classification of the conjugacy classes of unipotent elements, which requires $p$ to be "good" prime. The result still holds in general, thanks to the extension of Bala-Carter Theorem due to Duckworth [4].

\section{The infinite families}

This section covers the remaining four infinite families $(G, M)$ where $M=N_{G}(H)$ and $(G, H)$ are as in the following table:

\begin{tabular}{ccc}
\hline$G$ & $H$ & Remarks \\
\hline $\mathrm{PSp}_{2 m}(q)$ & $\Omega_{2 m}^{-}(q)$ & $m, q$ even \\
$\mathrm{P} \Omega_{2 m+1}(q)$ & $\Omega_{2 m}^{-}(q)$ & $m$ even, $q$ odd \\
$\mathrm{P} \Omega_{2 m}^{+}(q)$ & $\Omega_{2 m-1}(q)$ & $m$ even \\
$\mathrm{PSp}_{4}(q)$ & $\mathrm{PSp}_{2}\left(q^{2}\right)$ & \\
\hline
\end{tabular}

Table 2: The cases (a)-(d) of Table 1.

The idea is to compare the exponents of the Sylow $p$-subgroups of $G$ and $H$ using the results in the previous section, and check $\exp (M)$ when $\exp (G) \neq \exp (H)$. The Sylow $p$-subgroups of $G$ and $H$ obtained with the constructions presented in the previous section will be called $S_{G}$ and $S_{H}$ respectively if there could be any ambiguity. Recall also the notation: if $p \nmid q$ then $e=\operatorname{ord}_{p}(q)$ and $q^{e}=1+p^{r} x$ with $(x, p)=1$.

Proposition 3.1. $\operatorname{PSp}_{4}(q)$ contains a subgroup with the same exponent if and only if the characteristic of the underlying field is different from 3. An example of such a subgroup is $\operatorname{PSp}_{2}\left(q^{2}\right) \rtimes C_{2}$.

Proof. Consider first $p$ odd, $p \nmid q$. Since $p \neq 2$, the degree of the groups is too small to require a wreath product in the construction of a Sylow $p$-subgroup: using the notation in 2.1.2, this means that they are of the form $G_{0}$ or $G_{0}^{2}$, so $\exp _{p}\left(\operatorname{PSp}_{4}(q)\right)=\exp _{p}\left(\operatorname{PSp}_{2}\left(q^{2}\right)\right)=p^{r}$.

Take now $p=2$ in odd characteristic. By $(7)$ we have that $\exp _{2}\left(\operatorname{PSp}_{4}(q)\right)=2^{a}$ with $2^{a} \| q^{2}-1$, and $\exp _{2}\left(\operatorname{PSp}_{2}\left(q^{2}\right)\right)=2^{b-1}$ with $2^{b} \| q^{4}-1$. But $q^{4}-1=\left(q^{2}-1\right)\left(q^{2}+1\right)$, so $b=a+1$.

Finally, if $p \mid q$ then by (6)

$$
\exp _{p}\left(\operatorname{PSp}_{4}(q)\right)= \begin{cases}\exp _{p}\left(\operatorname{PSp}_{2}\left(q^{2}\right)\right) & \text { if } p \neq 2,3 \\ p \cdot \exp _{p}\left(\operatorname{PSp}_{2}\left(q^{2}\right)\right) & \text { if } p=2,3\end{cases}
$$

This means that the only problem is when the characteristic of the field is 2 or 3 , so we consider the normalizer $\operatorname{PSp}_{2}\left(q^{2}\right) \rtimes C_{2}$, which is a maximal subgroup of $\operatorname{PSp}_{4}(q)$.

If $q=3^{k}$, clearly $\exp \left(\mathrm{PSp}_{2}\left(q^{2}\right)\right)=\exp \left(\mathrm{PSp}_{2}\left(q^{2}\right) \rtimes C_{2}\right)=\frac{1}{3} \exp \left(\mathrm{PSp}_{4}(q)\right)$.

If $q=2^{k}$ we have $\exp \left(\mathrm{PSp}_{2}\left(q^{2}\right) \rtimes C_{2}\right)=2 \cdot \exp \left(\mathrm{PSp}_{2}\left(q^{2}\right)\right)=\exp \left(\mathrm{PSp}_{4}(q)\right)$. Indeed, consider the automorphism of $\mathbb{F}_{q^{2}}$ of order 2 given by $\sigma: x \mapsto x^{2^{k}}$. The action of $C_{2}$ onto $\operatorname{PSp}_{2}\left(q^{2}\right)$ is the induced automorphism, which maps each entry of the matrix with its $2^{k}$-th power.

Recall that the upper unitriangular matrices form a Sylow 2-subgroup and take $\alpha \in \mathbb{F}_{q^{2}}$, so that $x=\left(\left(\begin{array}{cc}1 & \alpha \\ 0 & 1\end{array}\right), \sigma\right) \in \operatorname{PSp}_{2}\left(q^{2}\right) \rtimes C_{2}$ has order 4 if $\alpha \neq 1$.

Proposition 3.2. $\operatorname{PSp}_{2 m}(q)$ with $m \geqslant 4$ even and $q$ even always contains a subgroup with the same exponent. An example of such a subgroup is $\Omega_{2 m}^{-}(q)$. 
Proof. Consider $p=2$ : by $(6), \exp _{2}\left(\operatorname{PSp}_{2 m}(q)\right)=\min \left\{2^{a} \mid 2^{a}>2 m-1\right\}$ and $\exp _{2}\left(\Omega_{2 m}^{-}(q)\right)=$ $\min \left\{2^{a} \mid 2^{a}>2 m-3\right\}$, so they're different iff $2 m-3<2^{a} \leqslant 2 m-1$ for some $a$. This would imply $2^{a-1}=m-1$, but $m$ is even and $m \geqslant 4$ so it can't occur.

Take now $p$ odd, then $S_{G}$ is isomorphic to a Sylow $p$-subgroup of $\operatorname{Sp}_{2 m}(q)$ and $S_{H}$ to a Sylow $p$-subgroup of $\mathrm{SO}_{2 m}^{-}(q)$. There are essentially two different situations, depending on $\left(p, q^{m}+1\right)$.

If $p \mid q^{m}+1$, a Sylow $p$-subgroup of $\mathrm{SO}_{2 m}^{-}(q)$ is isomorphic to a Sylow $p$-subgroup of $\mathrm{SO}_{2 m+1}(q)$ :

- if $e$ is even, $S_{H}$ and $S_{G}$ are Sylow $p$-subgroups of $\mathrm{GL}_{2 m+1}(q)$ and $\mathrm{GL}_{2 m}(q)$, respectively, so they are isomorphic, otherwise we would have $\exp _{p}\left(\mathrm{GL}_{2 m+1}(q)\right)<\exp _{p}\left(\mathrm{GL}_{2 m}(q)\right)$. Indeed, notice that $\left|\mathrm{GL}_{2 m+1}(q)\right|=\left|\mathrm{GL}_{2 m}(q)\right| \cdot\left(q^{2 m+1}-1\right)$, but $e$ is even so $p \nmid q^{2 m+1}-1$, and the order of their Sylow $p$-subgroups is the same;

- if $e$ is odd, $S_{H}$ is isomorphic to a Sylow $p$-subgroup of $\operatorname{Sp}_{2 m}(q)$.

If $p \nmid q^{m}+1$, a Sylow $p$-subgroup of $\mathrm{SO}_{2 m}^{-}(q)$ is isomorphic to a Sylow $p$-subgroup of $\mathrm{SO}_{2 m-1}(q)$ :

- if $e$ is even, $S_{H}$ and $S_{G}$ are Sylow $p$-subgroups of $\mathrm{GL}_{2 m-1}(q)$ and $\mathrm{GL}_{2 m}(q)$, respectively. Since $\left|\mathrm{GL}_{2 m}(q)\right|=\left|\mathrm{GL}_{2 m-1}(q)\right| \cdot\left(q^{2 m}-1\right)$, they have the same $p$-power part in the exponent, unless $p \mid q^{m}-1$ and $2 m=e p^{i}$ (see (1)). This can't happen, since $p \mid q^{m}-1$ implies $e \mid m$, then $2(m / e)=p^{i}$ but $p$ is odd;

- if $e$ is odd, $S_{H}$ is a Sylow $p$-subgroup of $\operatorname{Sp}_{2 m-2}(q)$. Like in the previous case, the exponent may be different if $2 m=2 e p^{i}$, but $m$ is even while both $e$ and $p$ are odd.

Proposition 3.3. $\mathrm{P} \Omega_{2 m+1}(q)$ with $m \geqslant 4$ even and $q$ odd always contains a subgroup with the same exponent, unless $p^{a}=2 m-1$ for some $a$, where $p$ is the characteristic of the underlying field. An example of such a subgroup is $\Omega_{2 m}^{-}(q)$.

Proof. $\exp _{2}\left(\mathrm{P} \Omega_{2 m+1}(q)\right)=\exp _{2}\left(\Omega_{2 m}^{-}(q)\right)$ follows immediately from (4) and (5).

Take now $p$ odd, $p \nmid q$. Since $p$ is odd, $S_{G}$ is isomorphic to a Sylow $p$-subgroup of $\mathrm{SO}_{2 m+1}(q)$ and $S_{H}$ to a Sylow $p$-subgroup of $\mathrm{SO}_{2 m}^{-}(q)$. If $p \mid q^{m}+1$, a Sylow $p$-subgroup of $\mathrm{SO}_{2 m}^{-}(q)$ is isomorphic to a Sylow $p$-subgroup of $\mathrm{SO}_{2 m+1}(q)$. If $p \nmid q^{m}+1$, it's isomorphic to a Sylow $p$-subgroup of $\mathrm{SO}_{2 m-1}(q)$.

If $e$ is odd, $S_{H}$ and $S_{G}$ are Sylow $p$-subgroups of $\operatorname{Sp}_{2 m-2}(q)$ and $\operatorname{Sp}_{2 m}(q)$, respectively, so they may not have the same exponent if $2 m=2 e p^{i}$, but $m$ is even while $e$ and $p$ are odd.

If $e$ is even, $S_{H}$ and $S_{G}$ are Sylow $p$-subgroups of $\mathrm{GL}_{2 m-1}(q)$ and $\mathrm{GL}_{2 m+1}(q)$, respectively. They may not have the same exponent if $2 m+1=e p^{i}+1$ (see (1)), but this can't happen:

- if $p\left|q^{m}-1, e\right| m$ and $p$ is odd;

- if $p \nmid q^{m}-1$, call $e=2 f$ so that $m=f p^{i}$ and $q^{2 f} \equiv 1 \bmod p$. Now $q^{m} \equiv q^{f p^{i}} \equiv q^{f} \equiv$ $\pm 1 \bmod p$, which means that either $p \mid q^{m}-1$ or $p \mid q^{m}+1$, contradiction.

Finally, consider $p \mid q$. By $(6), \exp _{p}\left(\mathrm{P} \Omega_{2 m+1}(q)\right)=\min \left\{p^{a} \mid p^{a}>2 m-1\right\}$ and $\exp _{p}\left(\Omega_{2 m}^{-}(q)\right)=$ $\min \left\{p^{a} \mid p^{a}>2 m-3\right\}$, so they're different iff $2 m-3<p^{a} \leqslant 2 m-1$, i.e. $p^{a}=2 m-1$, for some $a$. If that happens, $\exp _{p}\left(\mathrm{P} \Omega_{2 m+1}(q)\right)=p \cdot \exp _{p}\left(\Omega_{2 m}^{-}(q)\right)$.

$N_{\mathrm{P} \Omega_{2 m+1}(q)}\left(\Omega_{2 m}^{-}(q)\right)=\mathrm{K}_{2 m}^{-}(q)$. Indeed, it's known that $\mathrm{GO}_{2 m}^{-}(q) \times \mathrm{GO}_{1}(q) \simeq \mathrm{GO}_{2 m}^{-}(q) \times C_{2}$ is a maximal subgroup of $\mathrm{GO}_{2 m+1}(q)$. Taking the kernel of the determinant and of the spinor norm, we get that $\mathrm{K}_{2 m}^{-}(q)=\Omega_{2 m}^{-}(q) .2$ is a maximal subgroup of $\Omega_{2 m+1}(q)=\mathrm{P} \Omega_{2 m+1}(q)$, and the only one containing $\Omega_{2 m}^{-}(q)$.

Since the formula in (6) depends only on the "type" of group, in this case orthogonal, then $\exp _{p}\left(\mathrm{~K}_{2 m}^{-}(q)\right)=\exp _{p}\left(\Omega_{2 m}^{-}(q)\right)$ for $p \mid q$, therefore $\mathrm{P} \Omega_{2 m+1}(q)$ doesn't contain a subgroup with the same exponent if $p^{a}=2 m-1$.

Proposition 3.4. $\mathrm{P} \Omega_{2 m}^{+}(q)$ with $m \geqslant 4$ even always contains a subgroup with the same exponent. An example of such a subgroup is $\Omega_{2 m-1}(q)$. 
Proof. If $q$ is odd, $\exp _{2}\left(\mathrm{P} \Omega_{2 m}^{+}(q)\right)=\exp _{2}\left(\Omega_{2 m-1}(q)\right)$ follows immediately from (4) and (5).

If $p \mid q, \exp _{p}\left(\mathrm{P} \Omega_{2 m}^{+}(q)\right)=\exp _{p}\left(\Omega_{2 m-1}(q)\right)$ follows from (6) since $c=2 m-2$ for both groups.

Take now $p$ odd, $p \nmid q$. Since $p$ is odd, $S_{G}$ is isomorphic to a Sylow $p$-subgroup of $\operatorname{SO}_{2 m}^{+}(q)$ and $S_{H}$ to a Sylow $p$-subgroup of $\mathrm{SO}_{2 m-1}(q)$. If $p \nmid q^{m}-1$, a Sylow $p$-subgroup of $\mathrm{SO}_{2 m}^{+}(q)$ is isomorphic to a Sylow $p$-subgroup of $\mathrm{SO}_{2 m-1}(q)$. If $p \mid q^{m}-1$, it's isomorphic to a Sylow $p$-subgroup of $\mathrm{SO}_{2 m+1}(q)$, and the situation is the same as in the proof of 3.3.

\section{Alternating Groups}

As described in [5], if the alternating group $A_{n}$ contains a subgroup $M$ with the same exponent, then $A_{k} \unlhd M \leqslant\left(S_{k} \times S_{n-k}\right) \cap A_{n}$. From now on, suppose $k \geqslant n-k$, i.e. $k \geqslant n / 2$. Our claim is

Proposition 4.1. The alternating group $A_{n}(n \geqslant 5)$ doesn't contain a subgroup with the same exponent iff either $n=10, n=p^{r}$ with $p$ odd prime, or $n=p_{f}+1$ where $p_{f}$ is a Fermat prime.

Consider $p \leqslant n$ odd, then $\exp _{p}\left(A_{n}\right)=p^{t}$, where $p^{t} \leqslant n<p^{t+1}$ : take for example a $p^{t}$-cycle.

If $n=p^{t}$ then $\exp _{p}(M) \leqslant \exp _{p}\left(S_{k} \times S_{n-k}\right)=\exp _{p}\left(S_{k}\right)<p^{t}$, since $k<n$. Conversely, if $n \neq p^{t}$ we can take $k=n-1$ and get $\exp _{p}\left(A_{n-1}\right)=\exp _{p}\left(A_{n}\right)$.

Consider then $p=2$. Since a $2^{t}$-cycle doesn't belong to $A_{n}$, an element of maximal even order needs at least another disjoint transposition, which implies that $\exp _{2}\left(A_{n}\right)=2^{t}$ where $2^{t}+2 \leqslant n<$ $2^{t+1}+2$. If $n \neq 2^{t}+2$ then $\exp _{2}\left(A_{n-1}\right)=\exp _{2}\left(A_{n}\right)$. Conversely, if $n=2^{t}+2$ then take $k=n-2$ and get $\exp _{2}\left(A_{n}\right)=\exp _{2}\left(\left(S_{n-2} \times S_{2}\right) \cap A_{n}\right)$.

Therefore:

- if $n \neq p^{r}$ and $n \neq 2^{r}+2$, then $\exp \left(A_{n}\right)=\exp \left(A_{n-1}\right)$;

- if $n=p^{r}$, then $A_{n}$ can't have a subgroup with the same exponent;

- if $n=2^{r}+2$ and $n \neq p^{s}+1$ for any $p$ odd, then $\exp \left(A_{n}\right)=\exp \left(\left(S_{n-2} \times S_{2}\right) \cap A_{n}\right)$;

- if $n=2^{r}+2$ and $n=p^{s}+1$ for some $p$ odd, then $A_{n}$ can't have a subgroup with the same exponent, since $M \leqslant\left(S_{n-2} \times S_{2}\right) \cap A_{n}$ and $M \leqslant A_{n-1}$ imply $M \leqslant A_{n-2}$, hence $\exp _{q}(M)<\exp _{q}\left(A_{n}\right)$ for both $q=2$ and $q=p$, as seen above.

In particular, $A_{n}$ doesn't contain a subgroup with the same exponent if and only if either $n=p^{r}$ with $p$ odd prime, or $n=2^{r}+2=p^{s}+1$ with $p$ odd prime.

This last condition is realized when $2^{r}+1=p^{s}$. If $s=1$, then $2^{r}+1$ is a prime number iff it's a Fermat prime $(r=1$ is irrelevant since $n \geqslant 5)$. If $s>1$, the only possibility is $r=p=3$ and $s=2$, i.e. $n=10$, thanks to Mihăilescu's theorem [7].

\section{Other groups}

This section covers all the remaining pairs $(G, M)$ deduced from [5]. Since here the possible $M$ are explicitly listed, they can be studied computationally to get the following result:

Proposition 5.1. Consider the pairs $(G, M)$ in Table 1 not labelled (a)-(e). The only pairs which have $\exp (G)=\exp (M)$ are the following:

$$
\left(\mathrm{HS}, \mathrm{M}_{22}\right), \quad\left(\mathrm{M}_{12}, \mathrm{M}_{11}\right), \quad\left(\mathrm{M}_{24}, \mathrm{M}_{23}\right) .
$$

Proof. Most of the cases can be easily computed (we used GAP), the results are shown in Table 3 below. Therefore HS, $\mathrm{M}_{12}, \mathrm{M}_{24}$ have a subgroup with the same exponent, and the others don't, since the listed subgroups are maximal.

Notice that some pairs $(G, M)$ aren't included because $M$ missing from the ATLAS database (i.e. there isn't an explicit set of generators to make computations with):

$$
\left(\mathrm{U}_{4}(2), 2^{4} \rtimes A_{5}\right), \quad\left(\mathrm{L}_{6}(2), 2^{5} \rtimes \mathrm{L}_{5}(2)\right), \quad\left(\mathrm{P} \Omega_{8}^{+}(2), 2^{6} \rtimes A_{8}\right) .
$$

They can all be excluded using the same argument: call $M=2^{k} \rtimes H$, then $\exp (G)=3 \cdot n \cdot \exp (H)$ where $n=1,2$. Then it's clear that $\exp (M)$ is also missing a factor 3 , since $M$ is obtained by taking the semidirect product of $H$ with a 2-group. 


\begin{tabular}{lrlr}
\hline$G$ & $\exp (G)$ & $M$ & $\exp (M)$ \\
\hline$A_{6}$ & 60 & $\mathrm{~L}_{2}(5)$ & 30 \\
$\mathrm{U}_{3}(3)$ & 168 & $\mathrm{~L}_{2}(7)$ & 84 \\
$\mathrm{U}_{3}(5)$ & 840 & $A_{7}$ & 420 \\
$\mathrm{U}_{4}(2)$ & 180 & $S_{6}$ & 60 \\
$\mathrm{U}_{4}(3)$ & 2520 & $\mathrm{~L}_{3}(4)$ & 420 \\
& & $A_{7}$ & 420 \\
$\mathrm{U}_{5}(2)$ & 3960 & $\mathrm{~L}_{2}(11)$ & 330 \\
$\mathrm{U}_{6}(2)$ & 27720 & $\mathrm{M}_{22}$ & 9240 \\
$\mathrm{PSp}_{4}(7)$ & 4200 & $A_{7}$ & 420 \\
$\mathrm{Sp}_{6}(2)$ & 2520 & $S_{8}$ & 840 \\
$\mathrm{P}_{8}^{+}(2)$ & 2520 & $A_{9}$ & 1260 \\
$G_{2}(3)$ & 6552 & $\mathrm{~L}_{2}(13)$ & 546 \\
${ }^{2} F_{4}(2)^{\prime}$ & 3120 & $\mathrm{~L}_{2}(25)$ & 780 \\
$\mathrm{M}_{11}$ & 1320 & $\mathrm{~L}_{2}(11)$ & 330 \\
$\mathrm{M}_{12}$ & 1320 & $\mathrm{M}_{11}$ & 1320 \\
$\mathrm{M}_{24}$ & 212520 & $\mathrm{M}_{23}$ & 212520 \\
$\mathrm{HS}_{\mathrm{McL}}$ & 9240 & $\mathrm{M}_{22}$ & 9240 \\
$\mathrm{Mo}_{2}$ & 27720 & $\mathrm{M}_{22}$ & 9240 \\
$\mathrm{Co}_{3}$ & 637560 & $\mathrm{M}_{23}$ & 212520 \\
\hline
\end{tabular}

Table 3: Exponent evaluation for the remaining cases of Table 1.

Acknowledgments. I would like to thank my supervisor, Professor Andrea Lucchini, who inspired this work and helped me finding some key references.

\section{References}

[1] T. C. Burness and E. Covato. "On the prime graph of simple groups". In: Bulletin of the Australian Mathematical Society 91.2 (Apr. 2015), pp. 227-240.

[2] R. Carter and P. Fong. "The Sylow 2-Subgroups of the Finite Classical Groups". In: Journal of Algebra 1.2 (Jan. 1964), pp. 139-151.

[3] E. Covato. "On boundedly generated subgroups of profinite groups". In: Journal of Algebra 406 (May 2014), pp. 20-45.

[4] W. E. Duckworth. "Unipotent Classes in the Classical Groups Parametrized by Subgroups". In: Beiträge zur Algebra und Geometrie 52.1 (Apr. 2011), pp. 13-27.

[5] M. W. Liebeck, C. E. Praeger, and J. Saxl. "Transitive Subgroups of Primitive Permutation Groups". In: Journal of Algebra (2000), pp. 291-361.

[6] A. Lucchini, M. Morigi, and P. Shumyatsky. "Boundedly generated subgroups of finite groups". In: Forum Mathematicum 24.4 (June 2012), pp. 875-887.

[7] P. Mihăilescu. "Primary Cyclotomic Units and a Proof of Catalan's Conjecture". In: Journal für die reine und angewandte Mathematik 572 (2004), pp. 167-195.

[8] D. M. Testerman. " $A_{l}$-type Overgroups of Elements of Order $p$ in Semisimple Algebraic Groups and the Associated Finite Groups". In: Journal of Algebra (1995), pp. 34-76.

[9] A. J. Weir. "Sylow $p$-Subgroups of the Classical Groups Over Finite Fields with Characteristic Prime to p". In: Proceedings of the American Mathematical Society 6.4 (Aug. 1955), pp. 529533.

[10] W. J. Wong. "Twisted Wreath Products and Sylow 2-Subgroups of Classical Simple Groups". In: Mathematische Zeitschrift 97.5 (1967), pp. 406-424. 